Article

\title{
Pilot-Aided Frame Synchronization in Optical OFDM Systems
}

\author{
Funmilayo B. Offiong ${ }^{1, *}$, Sinan Sinanović ${ }^{2}\left(\mathbb{D}\right.$ and Wasiu O. Popoola ${ }^{3}$ \\ 1 Department of Electronic and Electrical Engineering, Obafemi Awolowo University, Ile-Ife 220005, Nigeria \\ 2 School of Engineering and Built Environment, Glasgow Caledonian University, Glasgow G4 0BA, UK; \\ sinan.sinanovic@gcu.ac.uk \\ 3 Institute for Digital Communications, University of Edinburgh, Edinburgh EH9 3JL, UK; \\ w.popoola@ed.ac.uk \\ * Correspondence: fboffiong@ieee.org
}

Received: 14 March 2019; Accepted: 27 April 2019; Published: 11 June 2020

check for updates

\begin{abstract}
Efficient frame synchronization is essential for data recovery in communication systems. In this study, a single pilot sequence is used to achieve both frame synchronization and peak-to-average power ratio (PAPR) reduction. The two systems considered are direct-current biased optical orthogonal frequency division multiplexing (DCO-OFDM) and asymmetrically clipped O-OFDM (ACO-OFDM). The pilot symbol is allocated to odd indexed subcarriers only. Thus, the synchronization algorithm leverages the mirror symmetric property of the pilot symbol within a frame to detect the start of the pilot signal at the receiver. This scheme has low complexity and gives precise frame synchronization at signal-to-noise ratios as low as $4 \mathrm{~dB}$ in an indoor visible light communication (VLC) channel.
\end{abstract}

Keywords: optical OFDM; pilot symbol; frame synchronization; symbol timing estimation; correlation-based method; optical wireless communication

\section{Introduction}

Synchronization at the receiver of a practical orthogonal frequency division multiplexing (OFDM) system design is one of the most crucial data detection stages that must be performed accurately in order to avoid system performance degradation due to symbol timing and carrier frequency offset [1]. A number of studies have been presented on synchronization techniques in both radio frequency OFDM (RF-OFDM) and optical OFDM (O-OFDM) systems [2,3]. The RF-OFDM synchronization schemes include the well-known training symbol-based method proposed by Schmidl and Cox in [2]. The timing metric of this method has a form of plateau as a result of the cyclic prefix concatenated to the OFDM symbols, thereby making it difficult to detect the precise start of the training symbol at the receiver. To reduce this ambiguity, Park et al. proposed a synchronization timing metric which has an impulse-like shape with a principal peak indicating the start of the training symbol [4]. Synchronization methods in O-OFDM include the modified versions of the RF-OFDM techniques to make them applicable to optical communication systems and a number of techniques specially designed for O-OFDM [5-9].

In optical communication system based on intensity modulation and direct detection (IM/DD) scheme, carrier frequency offset is not an issue because the O-OFDM signal is baseband [10]. Hence, the synchronization problems in the IM/DD O-OFDM receivers are due to symbol frame timing offset and sampling clock frequency offset [10]. Sampling clock frequency offset may occur when there is a mismatch between the clock frequency of the digital-to-analog converter (DAC) at the transmitter and analog-to-digital converter (ADC) at the receiver. Precise O-OFDM frame 
synchronization is important to avoid the symbol timing offset that may occur when the start of the O-OFDM symbol detected at the receiver either leads or lags behind the original transmitted O-OFDM symbol. Hence, proper symbol synchronization is required before demodulation in order to align the fast Fourier transform (FFT) window at the correct position within the transmitted sequence of each received O-OFDM symbol samples.

Our previous studies proposed and demonstrated the use of a pilot signal for peak-to-average power ratio (PAPR) reduction in pilot-assisted (PA) O-OFDM [11,12]. The PA technique embeds the pilot signal in the original O-OFDM signal for the purpose of PAPR reduction at the transmitter. The PA technique is based on data symbol phase rotation with several iterations of a randomly generated pilot symbol sequence in the frequency domain prior to inverse fast Fourier transform (IFFT) operation to generate the time domain signal. The phase rotation makes the data symbols statistically quasi-independent in order to reduce the probability of having subcarriers adding up coherently to produce high peaks. Thereafter, the PA time domain O-OFDM frame with the pilot phase sequence that gives the minimum PAPR is selected for transmission. The empirical evaluation of the PA PAPR reduction technique in a real indoor optical wireless communication (OWC) channel also shows the usefulness of the transmitted pilot signal for channel estimation at the receiver [12]. Furthermore, full use can still be made of the transmitted pilot symbol by utilizing it for symbol synchronization at the PA O-OFDM receiver.

In this paper, we present a frame synchronization technique in the PA O-OFDM system. The PA $\mathrm{O}-\mathrm{OFDM}$ frame consists of data and pilot symbols where a cluster of $U$ complex data symbols is allocated to subcarriers using the direct-current biased O-OFDM (DCO-OFDM) or asymmetrically clipped O-OFDM (ACO-OFDM) scheme. However, the randomly generated pilot sequence is allocated to odd indexed subcarriers only while even indexed subcarriers are set to zero in both schemes. We propose a frame synchronization timing metric based on the use of the mirror symmetry in the time domain pilot symbol and disparity between the average power of the O-OFDM symbols and pilot symbol to estimate the start of a PA O-OFDM frame. The pilot symbol is inserted within each frame since the symbol timing needs to be periodically monitored to ensure the transmitter and receiver are synchronized. The synchronization algorithm has low complexity and does not require prior knowledge of the transmitted pilot symbol at the receiver. In comparison with the modified Park's synchronization method [5,9], our proposed PA synchronization scheme has additional capability of using the pilot signal for PAPR reduction in O-OFDM transmitter and the timing metric has relatively low complexity with the ability to give precise synchronization at signal-to-noise ratio (SNR) as low as $4 \mathrm{~dB}$ in an indoor VLC channel.

The rest of the paper is structured as follows: description of the proposed frame synchronization algorithm is presented in Section 2. The simulation results and discussions on the performance of the proposed PA frame synchronization timing metric in the optical communication channel are presented in Section 3. Finally, concluding remarks are given in Section 4.

\section{Frame Synchronization in a Pilot-Assisted Optical OFDM System}

Transmitted OFDM signals usually have preamble/header and payload. Thus, one approach used in categorizing synchronization methods in OFDM is based on the portion of the transmitted signal utilized for the purpose of symbol timing offset estimation at the receiver. The two broad classifications under this category are called blind and preamble-based techniques [5]. The blind techniques exploit the unique properties of the transmitted OFDM payload to perform synchronization. For instance, the blind technique in [8] leverages the time domain symmetry in ACO-OFDM to achieve symbol timing synchronization at the receiver. On the other hand, the preamble-based techniques involve the use of predefined synchronization sequence appended in the frequency or time domain. A preamble-based method in [5] utilizes the switching signs pattern of a time domain training sequence to perform synchronization in order to reduce the computational complexity in O-OFDM system. Another approach of categorizing the synchronization methods is based on the type of operations 
performed by the synchronization algorithm at the receiver. This category includes correlation and cross-correlation techniques. Cross-correlation methods require prior knowledge of the transmitted synchronization sequence at the receiver in order to make a comparison between such a sequence and the received signal [3]. The correlation methods simply perform synchronization by searching for a portion of the received signal that best satisfies specific criteria of the timing metric correlation term [6]. The vital criteria for preamble used for synchronization timing in O-OFDM include ability to generate the preamble using the same hardware utilized for the O-OFDM data symbols, ability to use the preamble for other signal detection stage like channel estimation and the preamble is expected to have low peak-to-average power ratio (PAPR) because of the performance degradation effects of high PAPR in O-OFDM. All these criteria are greatly influenced by the intended applications.

The PA frame synchronization presented in this work is a correlation-based method that utilizes preamble called the pilot symbol. The PA frame has $U$ clusters of data symbols, $\mathbf{X}_{s}^{u}, u=1,2, \ldots, U$ and randomly generated pilot sequence, $\mathbf{X}_{p}$, as illustrated in Figure 1a,b for DCO-OFDM and ACO-OFDM respectively. The clusters of data symbols, $\mathbf{X}_{s}^{u}$, are drawn from $M$-QAM (M-ary quadrature amplitude modulation) constellation. The pilot symbol in both cases is generated using the IFFT by modulating odd indexed subcarriers only while zeros are used on the even indexed subcarriers, that is, $\mathbf{X}_{p}(i)=0$ for even $i$ where $i=0,1, \ldots, N-1$ and $N$ is the IFFT size. The real-valued time domain signal required in the IM/DD optical communication system is obtained by constraining the frequency domain subcarriers of the PA frame to have Hermitian symmetry. For the purpose of PAPR reduction, the phase of the data symbols is rotated with $R$ iterations of pilot symbol sequence. Thereafter, the IFFT output that gives the minimum PAPR value is selected for further processing. The PAPR reduction capability of the PA technique in O-OFDM with a complementary cumulative distribution function (CCDF) of $10^{-3}$ and $R=5$ is about $2.2 \mathrm{~dB}$. The process of activating odd indexed subcarriers only of the pilot symbol in this work is similar to the concept outlined in [2], while a novel timing metric algorithm for the PA frame synchronization at the receiver is introduced.

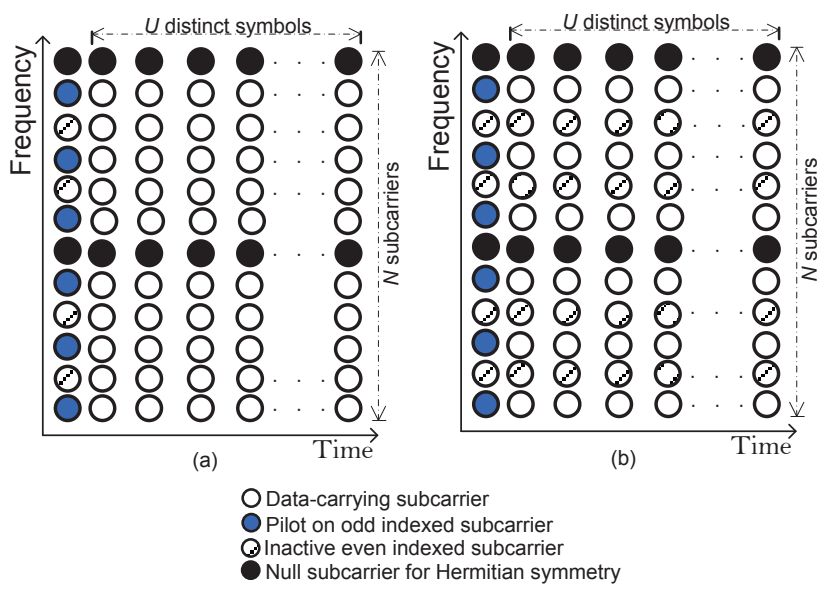

Figure 1. An illustration of a pilot-assisted (PA) frame in (a) direct-current biased optical orthogonal frequency division multiplexing (DCO-OFDM) and (b) asymmetrically clipped O-OFDM (ACO-OFDM).

The resulting time domain pilot symbol, $\mathbf{p}$, after the IFFT operation is given as:

$$
\mathbf{p}=\left[\begin{array}{lll}
p(0) & \mathbf{p}_{N / 2-1} & p(N / 2) \\
\mathbf{p}_{N / 2-1}^{\text {mirror }}
\end{array}\right]
$$

where $\mathbf{p}_{N / 2-1}$ represents samples of length $N / 2-1$ and $\mathbf{p}_{N / 2-1}^{\text {mirror }}$ represents mirror symmetry of $\mathbf{p}_{N / 2-1}, p(0)$ is the first pilot sample and $p(N / 2)$ is the $N / 2^{\text {th }}$ pilot sample. The mirror symmetry implies that $p(n)=p(N-n)$ for $n=1,2, \ldots N / 2-1$. To generate a time domain pilot signal with these properties using an IFFT with Hermitian symmetry, $\mathbf{X}_{p}(i)$, which is also used for data symbol phase rotation for the purpose of PAPR reduction, has its amplitude constrained to unity in order to 
preserve the electrical power of the data signals, while its phase can either be 0 or $\pi$ so as to maintain the original constellation of the input data symbols [11]. The mirror symmetry implies that, $\Delta_{\mathrm{PA}}$, which is the summation of the differences between the first half samples and mirror image of the second half samples of the pilot symbol, is:

$$
\begin{aligned}
\Delta_{\mathrm{PA}} & =\sum_{n=1}^{N / 2-1} p(n)-p(N-n) \\
& =0 .
\end{aligned}
$$

It should be noted that $p(0)$ and $p(N / 2)$ are excluded from the evaluation of $\Delta_{\mathrm{PA}}$ for the definition in (2) to hold. After adding cyclic prefix to the pilot symbol, the resulting symbol is given as:

$$
\mathbf{p}_{\mathrm{cp}}=\left[\begin{array}{lllll}
\mathbf{p}_{c p}^{\text {mirror }} & p(0) & \mathbf{p}_{N / 2-1} & p(N / 2) & \mathbf{p}_{N / 2-1}^{\text {mirror }}
\end{array}\right],
$$

where $c p$ is the length of the appended cyclic prefix and $\mathbf{p}_{c p}^{\text {mirror }}$ represents the last $c p$ samples of $\mathbf{p}_{N / 2-1}^{\text {mirror }}$. To obtain the unipolar signal required for IM, the time domain O-OFDM symbols are clipped at zero level in ACO-OFDM while adequate DC bias is added in DCO-OFDM.

At the receiver, the proposed timing synchronization algorithm exploits the mirror symmetric property of the received pilot signal, according to (2), to determine the start of each pilot symbol within the PA O-OFDM frames. The frame synchronization scheme is correlation-based with a detection window of size $N$ that slides through the received signal, $y(m)$. The start of the pilot signal within a frame is found by searching $y(m)$ for an O-OFDM symbol of $N$ samples in which the first half is identical to the mirror image of the second half using the proposed synchronization timing metric given as:

$$
P_{\mathrm{PA}}(\tau)=\sum_{m=\tau+1}^{\frac{N}{2}+\tau} \mathbf{y}(m)-\mathbf{y}(N-m),
$$

where $\tau$ is the PA timing metric sample index corresponding to the first sample in a window of $N$ samples. The proposed PA timing metric in (4) has low computational complexity because it requires simple addition operation. The receiver uses the minimum point of $P_{\mathrm{PA}}(\tau)$ to indicate the start of a pilot symbol.

In this work, the performance of the proposed PA method is compared with the Park's synchronization technique modified for O-OFDM system $[4,5,9]$. The modified Park's time domain training symbol sequence, after concatenation of cyclic prefix, is given as [5,9]:

$$
\mathbf{s}_{\mathrm{cp}}=\left[\mathbf{C}_{\mathrm{cp}} 0 \mathbf{C}_{N / 4-1} 0-\mathbf{C}_{N / 4-1}^{\text {mirror }} 0-\mathbf{C}_{N / 4-1} 0 \mathbf{C}_{N / 4-1}^{\text {mirror }}\right] \text {, }
$$

where $\mathbf{C}_{N / 4-1}$ represents samples of length $N / 4-1, \mathbf{C}_{N / 4-1}^{\text {mirror }}$ represents mirror symmetry of $\mathbf{C}_{N / 4-1}$ and $\mathbf{C}_{\mathrm{cp}}$ is the last $c p$ samples of $\mathbf{C}_{N / 4-1}^{\text {mirror }}$. The timing metric is given as [9]:

$$
M_{\mathrm{P}}(d)=\frac{1}{N / 4-1} \sum_{m=1}^{N / 4-1} r(d+m) r(d-m)
$$

where $d$ is the Park's timing metric sample index corresponding to the $N / 4^{\text {th }}$ sample in a window of $N / 2-1$ samples.

\section{Results and Discussions}

In this Section, we present the performance of the proposed PA frame synchronization timing metric by evaluating the average of the metric over 1000 PA O-OFDM frames. The performance evaluation is done in the presence of additive white Gaussian noise (AWGN) and indoor VLC channel $[13,14]$. The channel consists of line-of-sight (LOS) and non-LOS (NLOS) components with 
the LOS components dominating and the channel impulse response is obtained from power delay profile in [13] and workplace with open office in [14]. The two O-OFDM frames considered are the DCO-OFDM and ACO-OFDM structure. In the simulations, the $M$-QAM average symbol energy is normalized to unity, such that $\mathrm{E}\left[\left|\mathbf{X}_{s}^{u}(i)\right|^{2}\right]=1$. Other parameters used in the simulation are indicated in the caption of each figure.

Figure 2 shows the normalized average of the timing metric (4) for a PA ACO-OFDM. The plot shows that the timing metric varies as the detection window slides through the received signal in order to estimate the start of a pilot symbol. The start of a pilot symbol corresponds to the minimum point of the timing metric. The minimum average value of the metric is expected to be equal to zero, according to (2), when the detection window correlates with the received pilot symbol. Although this value of zero is not obtainable due to the effects of noise and indoor VLC channel on the received pilot signal, the proposed PA frame synchronization timing metric still results in a relatively large difference between the value at the start of a pilot symbol and other points within a frame. Figure 3 shows the normalized average of the modified Park's timing metric in ACO-OFDM with cyclic prefix length of $N / 8$. The correct location of the training symbol corresponds to the maximum point of the normalized average of the timing metric. To quantify the difference between the principal peak and the next peak, let $\beta=\left|\beta_{\mathrm{P}}-\beta_{2}\right|$, where $\beta_{\mathrm{P}}$ is the principal peak and $\beta_{2}$ is the second corresponding peak. Based on the pattern of the training symbol according to (5) and the sum of products operation used in the timing metric (6), a cyclic prefix whose length is greater or close to $N / 8$ results into principal and second corresponding peaks that are almost similar. Comparing Figures 2 and 3 where $c p=N / 8$ is utilized for ACO-OFDM in both cases, it is shown that the pattern of the time domain pilot symbol in (3) and the PA frame timing metric (4) result in $\beta=0.3$, whereas $\beta=0.04$ is obtained using the modified Park's method. This approach shows the robustness of the proposed PA synchronization algorithm to the effect of cyclic prefix length considered on the timing metric. The PA frame synchronization method also performs well for different ranges of $N$ and $U$.

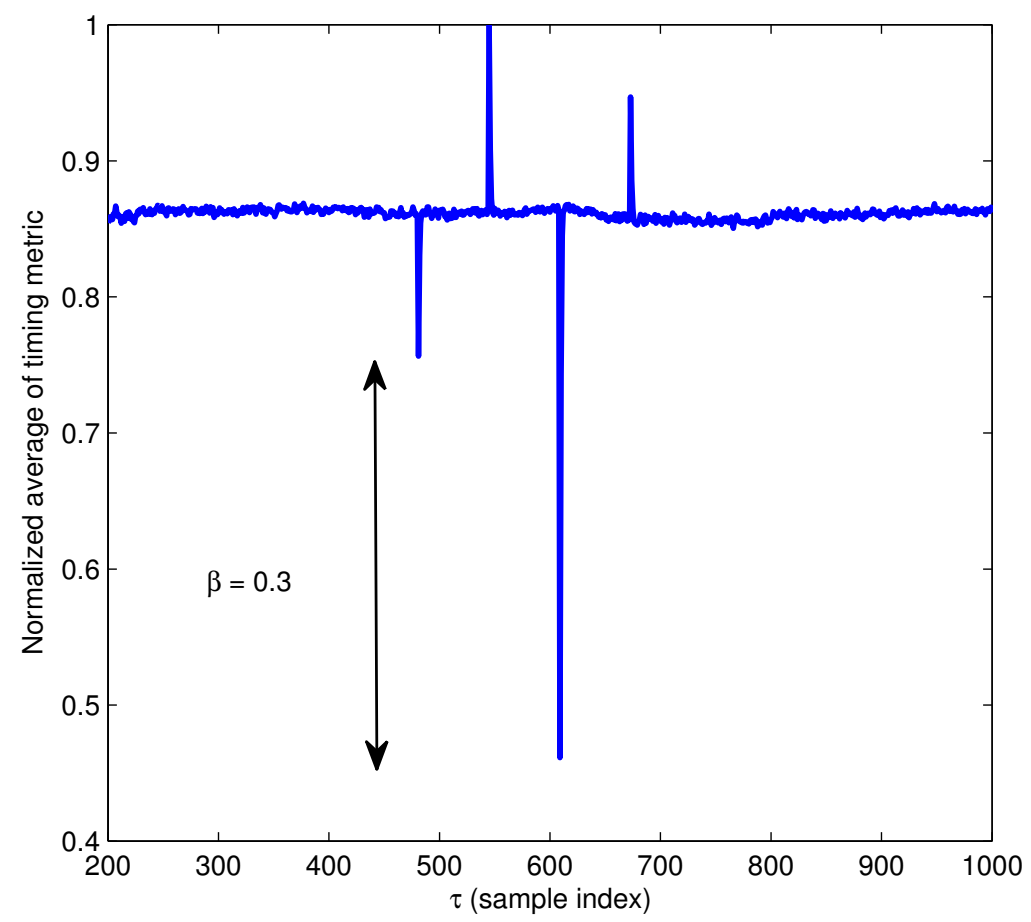

Figure 2. Plot of normalized average of the proposed timing metric (4) for a PA ACO-OFDM with $N=256, U=5, c p=N / 8$, signal-to-noise ratio (SNR) $=10 \mathrm{~dB}$, and 4-quadrature amplitude modulation (QAM). 


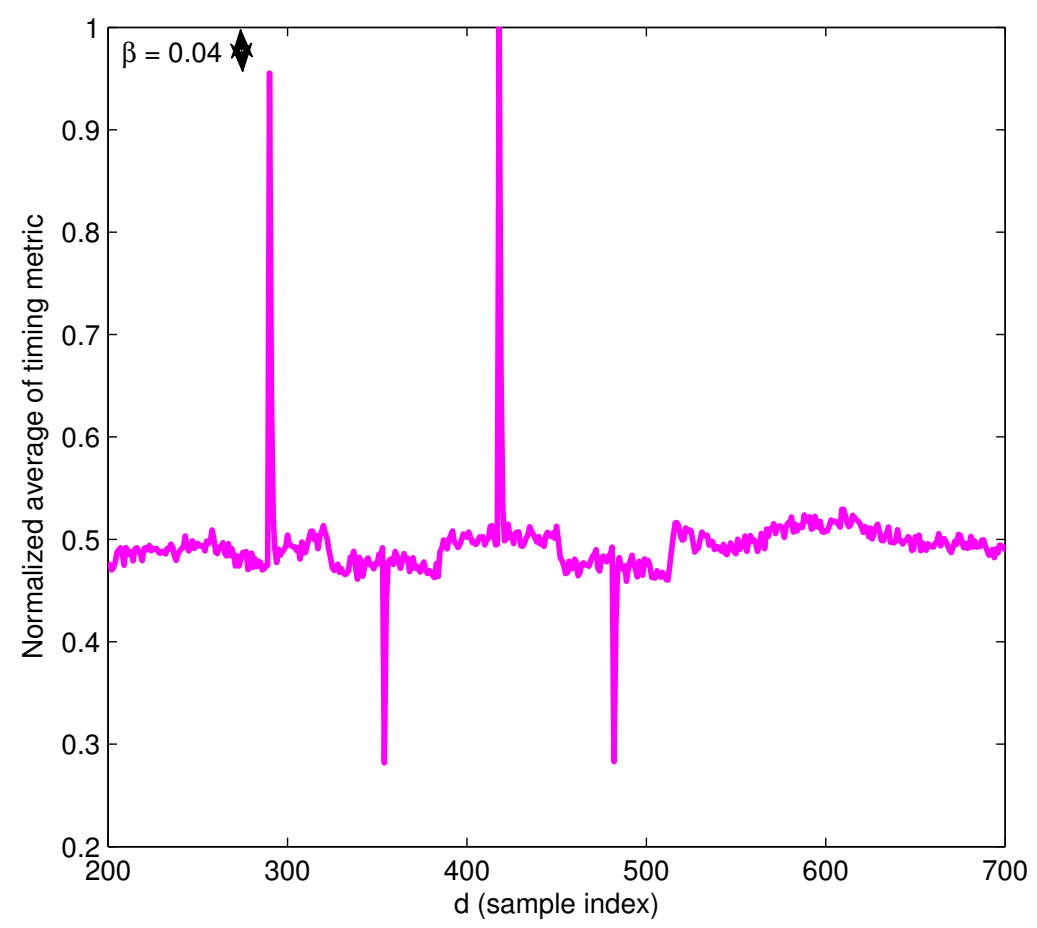

Figure 3. Plot of normalized average of the modified Park's timing metric (6) for an ACO-OFDM system with $N=256, U=5, c p=N / 8, \mathrm{SNR}=10 \mathrm{~dB}$, and 4-QAM.

In addition, the PA method has the lowest requirements in terms of computational complexity with respect to number of operations. For a pilot symbol with window size of $N$ samples, the PA timing metric (4) requires $N-3$ additions and no multiplication. The equivalent number of additions and multiplications in (6) is $\frac{N}{2}-8$ and $\frac{N}{4}+2$ respectively for preamble of a detection window of $\frac{N}{2}-1$ samples.

To investigate the rate of deviation of the PA frame synchronization timing metric (4) and modified Park's timing metric (6) from the exact symbol position, Figure 4 shows the mean of the timing offset in DCO-OFDM and ACO-OFDM for different SNR levels. In this work, a frame timing is considered to be estimated precisely when the timing metric gives its principal peak at the start of a pilot or training symbol without any tolerance margin. It is shown that the estimated start of a pilot symbol in DCO-OFDM and ACO-OFDM are at the precise timing point at SNR $\geq 4 \mathrm{~dB}$ and SNR $\geq 8 \mathrm{~dB}$ respectively using the PA method in both channel model considered. However, there is an average of the timing offset of about 2 and 217 samples in DCO-OFDM and ACO-OFDM respectively at $\mathrm{SNR}=2 \mathrm{~dB}$. In comparison with the modified Park's method, results show that the timing metric (6) is unable to achieve precise synchronization for the range of SNR considered. The metric has mean offset floor of about 165 and 265 samples in ACO-OFDM and DCO-OFDM, respectively. The performance plot shows the robustness of the PA frame synchronization timing metric in the presence of AWGN and indoor VLC channel. 


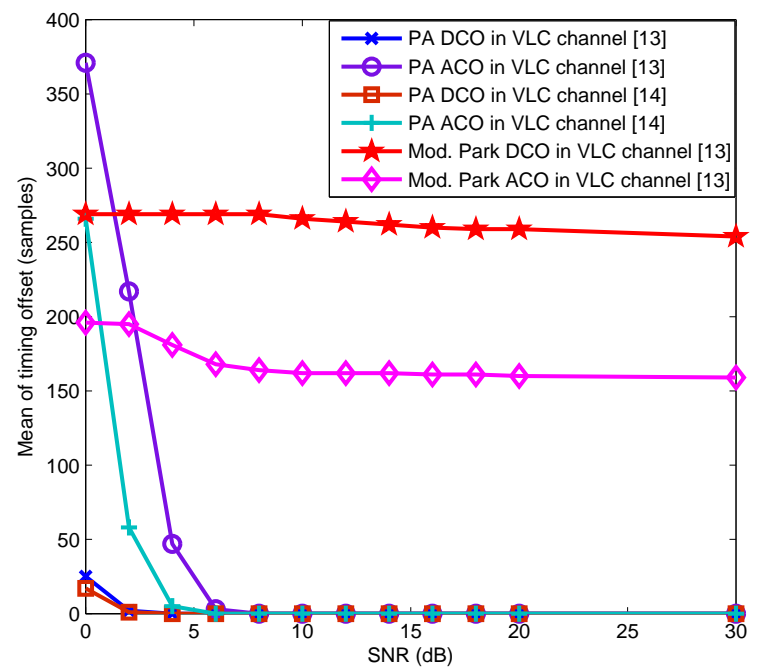

Figure 4. Mean of the timing offset in DCO-OFDM and ACO-OFDM using 1000 frames with $N=256$, $U=5, c p=N / 16$, and 4-QAM.

Figure 5 shows the CCDF plot of PAPR to illustrate the capability of using the pilot sequence utilized for frame synchronization to achieve PAPR reduction. The PAPR CCDF of PA ACO-OFDM and DCO-OFDM signal using $U=5, R=5,4$-QAM, 127 active subcarriers and an oversampling factor of 4 is shown in Figure 5. This figure also presents the plots of the basic ACO-OFDM and DCO-OFDM counterpart with no PAPR reduction technique for comparison. The result for the basic DCO-OFDM shows that 1 out of every $10^{3}$ DCO-OFDM frames has its PAPR greater than $14.1 \mathrm{~dB}$. However, the PA technique is capable of minimizing this threshold value such that 1 frame has its PAPR greater than $11.9 \mathrm{~dB}$ out of every $10^{3}$ frames. This implies a PAPR reduction gain of about $2.2 \mathrm{~dB}$ at CCDF of $10^{-3}$. Similarly, about the same PAPR reduction gain of $2.2 \mathrm{~dB}$ at CCDF of $10^{-3}$ is obtained when the PA technique is applied to ACO-OFDM system. Further comparison of the PA ACO-OFDM and DCO-OFDM with their basic counterparts shows that the PAPR reduction gain increases as CCDF decreases using the PA technique. The high signal peaks reduction capability of the PA technique implies that the technique can be implemented in O-OFDM system utilizing power-limited optical sources to ensure the transmitted signal is less susceptible to clipping due to the limited dynamic range of optical source.

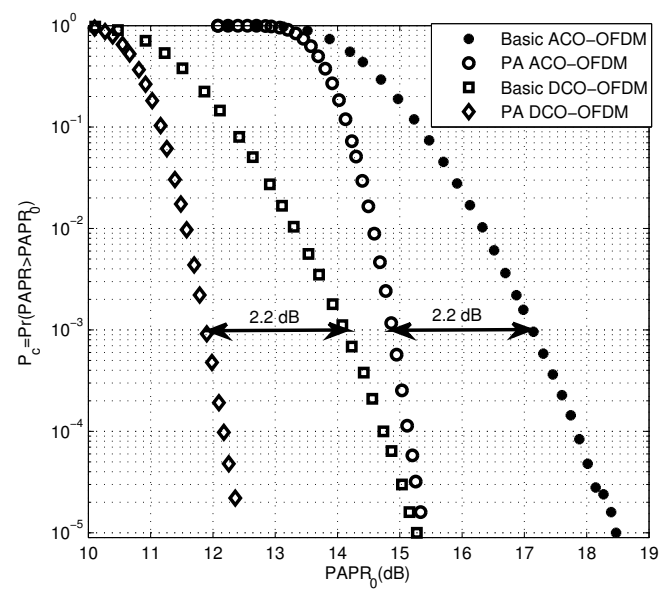

Figure 5. The complementary cumulative istribution function (CCDF) plot illustrating the peak-to-average power ratio (PAPR) reduction capability of PA technique in ACO-OFDM and DCO-OFDM using $U=5,4$-QAM and 127 active subcarriers (Pc is the probability that PAPR exceeds a particular defined value of $\mathrm{PAPR}_{0}$ ). 


\section{Conclusions}

In this paper, a frame synchronization algorithm is formulated in the PA O-OFDM system. The PA system utilizes a pilot signal that can be used for PAPR reduction at the transmitter and channel estimation at the receiver of an O-OFDM system. The proposed timing metric uses the mirror symmetric property of the received pilot signal without requiring prior knowledge of other system parameters for frame synchronization at the receiver. This metric has low computational complexity when compared with the Park's timing metric modified for O-OFDM because it requires only simple addition operation. The pattern of the time domain pilot symbol makes the method robust to the effect of cyclic prefix length on the timing metric such that it gives a distinct principal peak at the start of pilot symbol. Results show that the proposed timing metric is capable of performing precise frame synchronization in an indoor VLC channel when SNR $\geq 4 \mathrm{~dB}$ and SNR $\geq 8 \mathrm{~dB}$ in DCO-OFDM and ACO-OFDM, respectively. However, the ability of the algorithm to give precise frame synchronization timing reduces when the SNR decreases.

Author Contributions: Conceptualization, F.B.O., S.S. and W.O.P.; Data curation, F.B.O., S.S. and W.O.P.; Formal analysis, F.B.O., S.S. and W.O.P.; Funding acquisition, S.S.; Investigation, F.B.O., S.S. and W.O.P.; Methodology, F.B.O., S.S. and W.O.P.; Project administration, S.S. and W.O.P.; Resources, F.B.O.; Software, F.B.O.; Supervision, S.S. and W.O.P.; Validation, F.B.O.; Visualization, F.B.O.; Writing original draft, F.B.O.; Writing review and editing, S.S. and W.O.P. All authors have read and agreed to the published version of the manuscript.

Funding: This research received no external funding.

Conflicts of Interest: The authors declare no conflict of interest.

\section{Abbreviations}

The following abbreviations are used in this manuscript:

$\begin{array}{ll}\text { ACO-OFDM } & \text { Asymmetrically Clipped Optical Orthogonal Frequency Division Multiplexing } \\ \text { ADC } & \text { Analog-to-Digital Converter } \\ \text { AWGN } & \text { Additive White Gaussian Noise } \\ \text { CCDF } & \text { Complementary Cumulative Distribution Function } \\ \text { DAC } & \text { Digital-to-Analog Converter } \\ \text { DCO-OFDM } & \text { Direct Current Optical Orthogonal Frequency Division Multiplexing } \\ \text { FFT } & \text { Fast Fourier Transform } \\ \text { IFFT } & \text { Inverse Fast Fourier Transform } \\ \text { IM/DD } & \text { Intensity Modulation and Direct Detection } \\ \text { LOS } & \text { Line-of-Sight } \\ \text { M-QAM } & \text { M-ary Quadrature Amplitude Modulation } \\ \text { N-LOS } & \text { Non-Line-of-Sight } \\ \text { OFDM } & \text { Orthogonal Frequency Division Multiplexing } \\ \text { O-OFDM } & \text { Optical Orthogonal Frequency Division Multiplexing } \\ \text { OWC } & \text { Optical Wireless Communications } \\ \text { PA } & \text { Pilot-Assisted } \\ \text { PAPR } & \text { Peak-to-Average Power Ratio } \\ \text { PA O-OFDM } & \text { Pilot-Assisted Optical Orthogonal Frequency Division Multiplexing } \\ \text { RF-OFDM } & \text { Radio Frequency Orthogonal Frequency Division Multiplexing } \\ \text { SNR } & \text { Signal-to-Noise Ratio }\end{array}$

\section{References}

1. Cho, Y.S.; Kim, J.; Yang, W.Y.; Kang, C.G. MIMO-OFDM Wireless Communications with MATLAB; John Wiley \& Sons: Hoboken, NJ, USA, 2010.

2. Schmidl, T.M.; Cox, D.C. Robust frequency and timing synchronization for OFDM. IEEE Trans. Commun. 1997, 45, 1613-1621. [CrossRef] 
3. Ranjha, B.A.; Kashani, M.A.; Kavehrad, M.; Deng, P. Robust timing synchronization for AC-OFDM based optical wireless communications. In Proceedings of the Integrated Communication, Navigation and Surveillance Conference (ICNS), Herndon, VA, USA, 21-23 April 2015; pp. 1-9.

4. Park, B.; Cheon, H.; Kang, C.; Hong, D. A novel timing estimation method for OFDM systems. IEEE Commun. Lett. 2003, 7, 239-241. [CrossRef]

5. Goroshko, K.; Manolakis, K.; Grobe, L.; Jungnickel, V. Low-latency synchronization for OFDM-based visible light communication. In Proceedings of the IEEE International Conference on Communication (ICC) Workshop, London, UK, 8-12 June 2015; pp. 1327-1332.

6. Truong, T.A.; Arzel, M.; Lin, H.; Jahan, B.; Jezequel, M. New low-complexity and robust time synchronization technique for optical IMDD OFDM transmissions. Opt. Express 2014, 22, 14322-14340. [CrossRef] [PubMed]

7. Jin, X.Q.; Tang, J.M. Optical OFDM synchronization with symbol timing offset and sampling clock offset compensation in real-time IMDD systems. IEEE Photonics J. 2011, 3, 187-196. [CrossRef]

8. Freda, M.M.; Murray, J.M. Low-complexity blind timing synchronization for ACO-OFDM-based optical wireless communications. In Proceedings of the IEEE Globecom Workshops, Miami, FL, USA, 6-10 December 2010; pp. 1031-1036.

9. Tian, S.; Panta, K.; Suraweera, H.A.; Schmidt, B.J.S.J.; McLaughlin, S.; Armstrong, J. A novel timing synchronization method for ACO-OFDM-based optical wireless communications. IEEE Trans. Wirel. Commun. 2008, 7, 4958-4967. [CrossRef]

10. Ghimire, B.; Stefan, I.; Elgala, H.; Haas, H. Time and frequency synchronisation in optical wireless OFDM networks. In Proceedings of the IEEE 22nd International Symposium on Personal, Indoor and Mobile Radio Communications, Toronto, ON, Canada, 11-14 September 2011; pp. 819-823.

11. Popoola, W.O.; Ghassemlooy, Z.; Stewart, B.G. Pilot-Assisted PAPR Reduction Technique for Optical OFDM Communication Systems. J. Lightw. Technol. 2014, 32, 1374-1382. [CrossRef]

12. Ogunkoya, F.; Popoola, W.; Shahrabi, A.; Sinanović, S. Performance Evaluation of Pilot-assisted PAPR Reduction Technique in Optical OFDM Systems. IEEE Photonics Technol. Lett. 2015, 27, 1088-1091. [CrossRef]

13. Kwonhyung, L.; Hyuncheol, P.; Barry, J.R. Indoor Channel Characteristics for Visible Light Communications. IEEE Commun. Lett. 2011, 15, 217-219.

14. Uysal, M.; Miramirkhani, F.; Narmanlioglu, O.; Baykas, T.; Panayirci, E. IEEE 802.15.7r1 Reference Channel Models for Visible Light Communications. IEEE Commun. Mag. 2017, 55, 212-217. [CrossRef]

(C) 2020 by the authors. Licensee MDPI, Basel, Switzerland. This article is an open access article distributed under the terms and conditions of the Creative Commons Attribution (CC BY) license (http://creativecommons.org/licenses/by/4.0/). 